\title{
Three-chromophore FRET microscopy to analyze multiprotein interactions in living cells
}

\author{
Emilia Galperin $^{1,2}$, Vladislav V Verkhusha ${ }^{1,2}$ \& Alexander Sorkin ${ }^{1}$
}

Nearly every major process in a cell is carried out by assemblies of multiple dynamically interacting protein molecules. To study multi-protein interactions within such molecular machineries, we have developed a fluorescence microscopy method called three-chromophore fluorescence resonance energy transfer (3-FRET). This method allows analysis of three mutually dependent energy transfer processes between the fluorescent labels, such as cyan, yellow and monomeric red fluorescent proteins. Here, we describe both theoretical and experimental approaches that discriminate the parallel versus the sequential energy transfer processes in the 3-FRET system. These approaches were established in vitro and in cultured mammalian cells, using chimeric proteins consisting of two or three fluorescent proteins linked together. The 3-FRET microscopy was further applied to the analysis of three-protein interactions in the constitutive and activation-dependent complexes in single endosomal compartments. These data highlight the potential of 3-FRET microscopy in studies of spatial and temporal regulation of signaling processes in living cells.

Many cellular processes are governed by multi-component molecular machineries that rely on dynamic and highly coordinated protein-protein interactions. For example, assembly of protein complexes during signal transduction processes increases the speed of enzymatic reactions, ensures the specificity of signaling and targets signaling molecules to proper intracellular compartments. During recent years, fluorescence resonance energy transfer (FRET) has become a key method for the analysis of proteinprotein interactions during signal transduction in living cells ${ }^{1-3}$. FRET studies using proteins tagged with mutant derivatives of the green fluorescent protein (GFP) have demonstrated the formation of complexes of signaling proteins in various intracellular compartments ${ }^{4-7}$. FRET-based genetically encoded biosensors for second messengers, protein phosphorylation and activity of small GTPases have provided insights into the spatial and temporal regulation of signaling processes ${ }^{8-12}$.

The combination of enhanced cyan (CFP) and yellow (YFP) fluorescent proteins has proven to be most effective in many FRET studies. Cloning of Anthozoa fluorescent proteins, such as red DsRed $^{13,14}$ and far-red $\mathrm{HcRed}^{15}$, has expanded the in vivo applications of FRET, but a major limitation of Anthozoa proteins for FRET applications is their obligate oligomerization ${ }^{16}$. Recent generation of a monomerized mutant of DsRed, monomeric red fluorescent protein $1(\mathrm{mRFP})^{17}$, provides the opportunity to generate functional monomeric fusion proteins and to use mRFP as a FRET acceptor with proteins fused to GFP or its mutants.

Various methods of FRET measurements have been used to visualize protein-protein interactions ${ }^{18}$. The general limitation of these methods is that they only permit the analysis of interactions between two proteins. To analyze multi-component signaling complexes, a method to measure interactions between several proteins in vivo is required. 3-FRET methods using organic dyes to study multiple interactions in vitro have recently emerged ${ }^{19-21}$. We have designed experimental conditions to use mRFP as a FRET acceptor paired with CFP or YFP. These experiments have led to the development of a method of 3-FRET that is capable of measuring FRET signals within a system of three donor-acceptor pairs, such as CFP-YFP, CFP-mRFP and YFP-mRFP in vitro and in vivo.

\section{RESULTS}

\section{CFP-YFP-mRFP 3-FRET in vitro}

To examine whether mRFP is an efficient acceptor of energy transfer from CFP and YFP, we expressed concatemeric proteins consisting of a tandem of either CFP and mRFP or YFP and mRFP, each containing the factor $\mathrm{X}_{\mathrm{a}}$ protease cleavage site within the linker between fluorescent proteins, in bacterial cells and purified them (Fig. 1a). Emission spectra were measured before and after digestion by factor $\mathrm{X}_{\mathrm{a}}$. Cleavage of fusion proteins led to an increase in donor emission and a simultaneous decrease in red emission of mRFP acceptor, indicative of FRET from CFP and YFP to mRFP (Fig. 1b-c). Calculation of FRET efficiencies (designated as E) based on the increase of donor fluorescence emission upon cleavage of the chimeric proteins produced $E_{\mathrm{CR}}=0.40$ and $E_{\mathrm{YR}}=0.45$ for CFP-mRFP and YFP-mRFP pairs, respectively. For comparison, $E_{C Y}$ for CFP-YFP pair was 0.42 (Fig. 1d and Supplementary Table 1 online), which is consistent with previously published data ${ }^{22}$.

To examine whether FRET between CFP, YFP and mRFP can be detected simultaneously, we prepared a triple-fusion protein consisting of CFP, YFP and mRFP (Fig. 1a). Within this construct parallel $\mathrm{CFP} \rightarrow \mathrm{YFP}$ and $\mathrm{CFP} \rightarrow \mathrm{mRFP}$ FRET, as well as a further

${ }^{1}$ Department of Pharmacology, University of Colorado Health Sciences Center, Aurora, Colorado 80045, USA. ${ }^{2}$ These authors contributed equally to this work. Correspondence should be addressed to A.S. (Alexander.Sorkin@uchsc.edu).

PUBLISHED ONLINE 18 NOVEMBER 2004; D0I:10.1038/NMETH720 

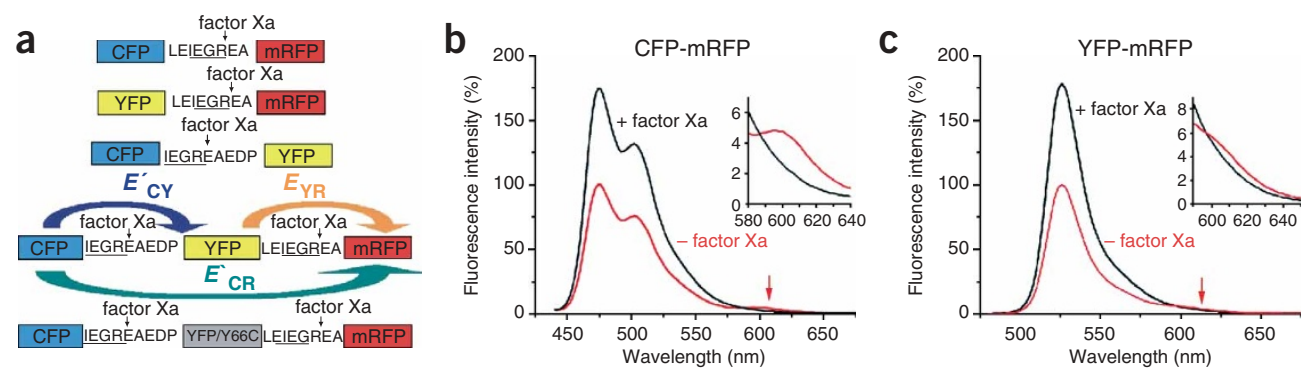

e

Figure 1 | Spectral analysis of CFP-YFP-mRFP fusion proteins in vitro. (a) The schematic representation of fusion concatemeric constructs CFP-mRFP, YFP-mRFP, CFP-YFP, CFP-YFP-mRFP and CFP-YFP/Y66C-mRFP. Black arrows indicate specific cleavage sites for the factor $X_{a}$ protease, which recognizes amino acid sequence IEGR (underlined). Parallel FRET from CFP to YFP ( $\left.E_{C Y}^{\prime}\right)$ and mRFP $\left(E_{C R}^{\prime}\right)$ and one-step FRET from YFP to mRFP $\left(E_{Y R}\right)$ observed in the CFP-YFP-mRFP fusion protein are shown by arrows. (b-f) Emission spectra before (red lines) and after (black lines) treatment with factor $X_{a}$ are presented for CFP-mRFP (b), YFP-mRFP (c), CFP-YFP (d), CFP-YFP-mRFP (e) and CFP-YFP/Y66C-mRFP (f) purified

proteins. The excitation was carried out at $420 \mathrm{~nm}(\mathbf{b}, \mathbf{d}, \mathbf{e}, \mathbf{f})$ or $470 \mathrm{~nm}(\mathbf{c})$.

The insets in the panels (b-f) zoom in the mRFP emission range. The sensitivity of spectrofluorimeter was substantially lower in the red region of the

fluorescence spectra, which resulted in low arbitrary fluorescence intensities measured for mRFP emission. The spectral changes observed upon cleavage of the fusion proteins are indicated by red arrows in $\mathbf{b}, \mathbf{c}, \mathbf{e}$ and $\mathbf{f}$.

one-step YFP $\rightarrow$ mRFP FRET, are possible. Separation of fluorescent proteins in the CFP-YFP-mRFP fusion resulted in an $85 \%$ increase of emission at $474 \mathrm{~nm}$, which yields total energy transfer from CFP of $0.46\left(E_{\mathrm{C} \rightarrow \mathrm{YR}}=0.46\right)$ (Fig. 1e). This value is higher than $E_{\mathrm{CY}}$ efficiency measured for the CFP-YFP fusion $\left(E_{\mathrm{CY}}=0.42\right)$ (Fig. 1d), suggesting that energy from CFP is transferred not only to YFP but also directly to mRFP in the triple-fusion protein. To calculate the efficiency of CFP $\rightarrow$ mRFP FRET in the CFP-YFPmRFP protein, we generated a control construct containing the YFP Y66C mutant lacking the chromophore (Fig. 1a). The CFPYFP/Y66C-mRFP fusion exhibited $E_{\mathrm{CR}}$ efficiency of 0.22 (Fig. 1f), which reflected the increase in distance between CFP and mRFP in the triple-fusion as compared with that in the CFP-mRFP doublefusion protein $\left(E_{\mathrm{CR}}=0.40\right)$.

The $E$ values for the two-chromophore constructs, which exhibit the same respective spatial locations of CFP, YFP and mRFP proteins as those in the CFP-YFP-mRFP chimera, were used to calculate apparent efficiencies of energy transfer from CFP to YFP $\left(E_{\mathrm{CY}}^{\prime}\right)$ and to mRFP $\left(E_{\mathrm{CR}}^{\prime}\right)$ in this three-chromophore construct (see mathematical description and calculations of 3-FRET efficiencies in Supplementary Methods online). These calculations yielded $E^{\prime}{ }_{\mathrm{CR}}=0.14$ and $E_{\mathrm{CY}}^{\prime}=0.36$; therefore, $E_{\mathrm{C} \rightarrow \mathrm{YR}}{ }^{\mathrm{THEOR}}=0.50$, which is in good agreement with the experimentally obtained value for $E_{\mathrm{C} \rightarrow \mathrm{YR}}{ }^{\mathrm{EXP}}=0.46$. Our calculation also showed that $\sim 45 \%$ of energy from CFP reaches $\mathrm{mRFP}$ directly, and the remaining $55 \%$ is the result of the sequential $\mathrm{CFP} \rightarrow \mathrm{YFP} \rightarrow \mathrm{mRFP}$ FRET. Thus, these experiments demonstrate that three mutually dependent FRET processes were observed in the CFP-YFP-mRFP fusion protein.

\section{CFP-YFP-mRFP 3-FRET in mammalian cells}

To develop live-cell mRFP-based FRET microscopy techniques, we expressed CFP-mRFP and YFP-mRFP fusion proteins (Fig. 1a) in
COS-1 cells. A three-filter method of sensitized FRET measurements ${ }^{23}$ was used, as described in our previous studies with the CFP-YFP pair ${ }^{7}$. Corrected FRET $\left(\right.$ FRET $^{\mathrm{C}}$ ) signals (equation 1A) were obtained for both YFP-mRFP and CFP-mRFP pairs (Fig. 2a,b). The FRET efficiency $(E)$ images, calculated according to equation 2, showed that $E$ was independent of the expression level of fluorescent proteins (Fig. 2a,b), indicating that concentration-driven, nonspecific FRET signals are not detected in our system.

To confirm the detection of FRET with mRFP acceptor by sensitized emission, we used a second approach of measuring FRET efficiencies, a method of donor fluorescence recovery after acceptor photobleaching (DFRAP) ${ }^{24}$. Because of rapid diffusion of the proteins in the cytosol, mRFP was photobleached in whole cells. There was a substantial increase in donor intensities after mRFP photobleaching, indicative of FRET (Fig. 2a,b). The FRET efficiencies $\left(E^{\mathrm{P}}\right)$ were calculated (equation 3 ) for whole cells rather than on a pixel-by-pixel basis owing to considerable redistribution of fluorescence intensities in the cell during photobleaching. The mean $E^{\mathrm{P}}$ values of YFP-mRFP (0.23) and CFP-mRFP FRET (0.14) in experiments where the maximal photobleaching was achieved $(>90 \%)$ were slightly lower than the FRET efficiencies obtained using sensitized emission measurements $(0.25$ and 0.17 , respectively), owing to incomplete photobleaching of the acceptor and, therefore, incomplete donor dequenching.

To examine whether microscopic analysis can be used to detect 3-FRET in living cells, we expressed the triple fusion CFP-YFPmRFP construct (Fig. 1a) in cells. We set up a six-filter channel method, which allowed acquisition of images of all three chromophores and three 'raw' FRET images. We used these six images to calculate three FRET $^{\mathrm{C}}$ images (equation 1A). Positive FRET ${ }^{\mathrm{C}}$ signals were detected for all three FRET pairs: CFP-YFP, 


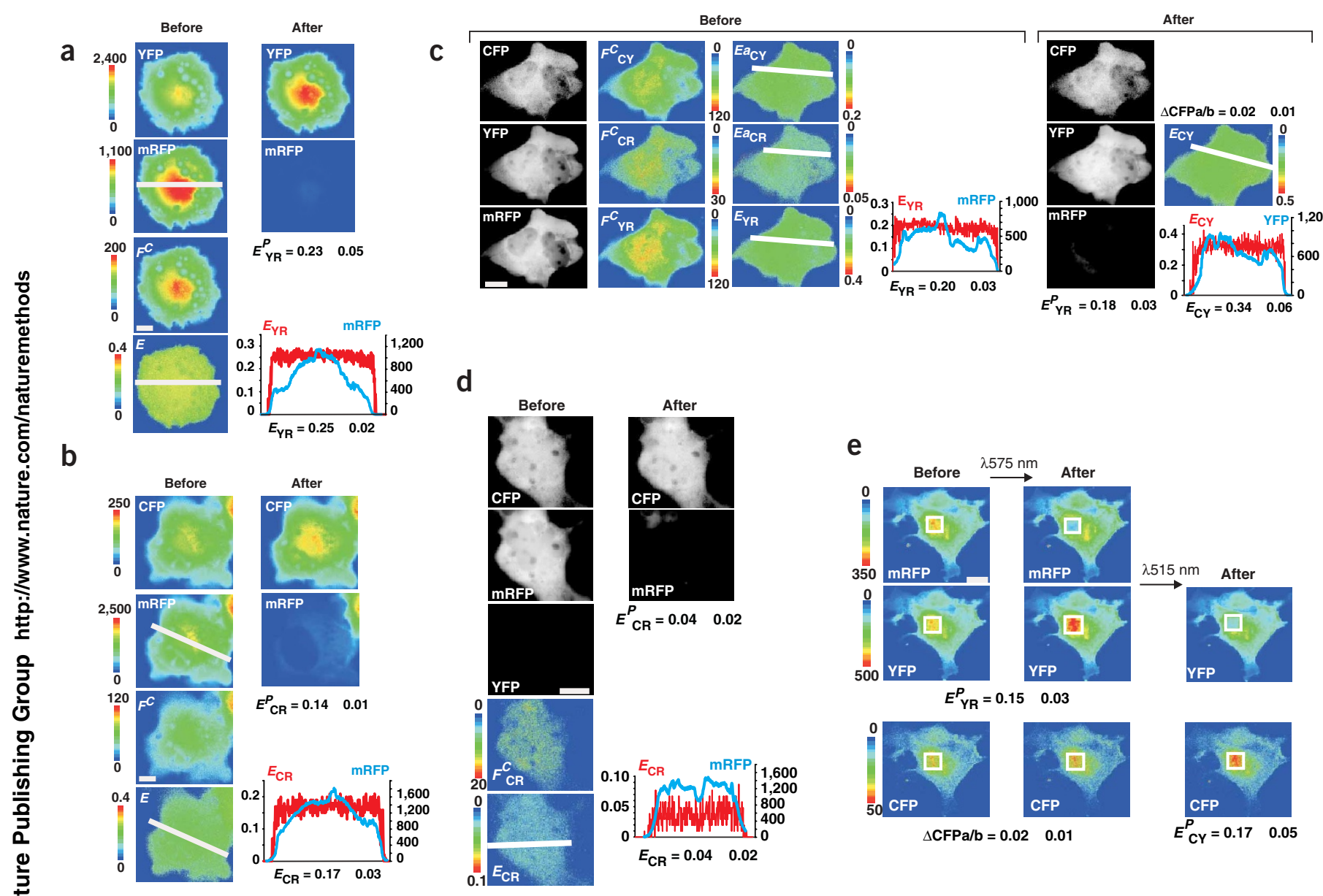

Figure 2 | Whole-cell 2-FRET and 3-FRET analysis in cells expressing CFP-YFP-mRFP concatemeric constructs. YFP-mRFP (a) or CFP-mRFP (b) fusion proteins (see Fig. 1a) were expressed in COS-1 cells for 2 d. Three images (donor, acceptor and FRET filter sets) were acquired from living cells before and after mRFP photobleaching. $\mathrm{FRET}^{\mathrm{C}}$ and $E$ images were calculated and presented in quantitative pseudo-color. On the graphs, the random linear intensity scans of $E$ (red) and mRFP images (blue) are presented. The mean values of $E$ and $E^{P}$ ( \pm s.d.) obtained using sensitized emission method and DFRAP are presented under corresponding images. (c) CFP-YFP-mRFP fusion protein was expressed for $2 \mathrm{~d}$. Six images (YFP, CFP, mRFP and three FRET images) were acquired from living cells before and after mRFP photobleaching at $575 \mathrm{~nm}$. FRET ${ }^{\mathrm{C}}$ and $E$ images were calculated and presented as in a. Linear intensity scans of $E$ (red) and acceptor images (blue) are presented. The mean $E$ and $E^{P}$ values of YFP-mRFP FRET were also calculated based on sensitized emission and donor dequenching, and presented under corresponding images. The mean $E$ value for CFP-YFP FRET was calculated after mRFP photobleaching. $\triangle \mathrm{CFPa} / \mathrm{b}$ is the fraction increase of CFP fluorescence intensity after mRFP photobleaching. (d) CFP-YFP/Y66C-mRFP fusion protein was expressed for $2 \mathrm{~d}$. Six images (YFP, CFP, mRFP and three FRET images) were acquired from living cells before and after mRFP photobleaching. FRET ${ }^{C}$ and $E$ images were calculated as described in a. Linear intensity scans of $E$ (red) and acceptor images (blue) are presented. The mean $E$ values ( \pm s.d.) of CFP-mRFP were calculated based on sensitized emission and donor dequenching. (e) Sequential photobleaching of mRFP and YFP in fixed cells expressing CFP-YFP-mRFP fusion protein as in c. The cells were fixed with freshly prepared paraformaldehyde $(4 \%)$. The photobleached region is indicated by a white rectangle. Mean FRET efficiencies $\left(E^{P-} \pm\right.$ s.d.) are presented. $\Delta$ CFPa/b is the fraction increase of CFP fluorescence intensity after mRFP photobleaching. All fluorescence intensity measurements in a-e are presented in arbitrary linear units. Each value represents an averaged value from 5-6 cells. Scale bar, $10 \mu \mathrm{m}$.

CFP-mRFP and YFP-mRFP (Fig. 1a). In this system, YFP-mRFP FRET does not depend on the presence of CFP. Therefore, the transfer efficiency $\left(E_{\mathrm{YR}}\right)$ images were directly calculated using FRET $^{\mathrm{YR}}$ and YFP images (Fig. 2c). The energy transfer from YFP to mRFP in the triple-fusion construct was confirmed by DFRAP (Fig. 2c). Elimination of mRFP by photobleaching allowed calculation of FRET efficiency $\left(E_{\mathrm{CY}}\right)$ images for a CFP-YFP pair (Fig. 2c).

As predicted from in vitro experiments (Fig. 1), CFP $\rightarrow$ mRFP FRET $^{\mathrm{C}}$ signals in the CFP-YFP-mRFP fusion result from direct $\mathrm{CFP} \rightarrow \mathrm{mRFP}$ and sequential $\mathrm{CFP} \rightarrow \mathrm{YFP} \rightarrow \mathrm{mRFP}$ FRET. Therefore, transfer efficiency values cannot be easily derived from $\mathrm{FRET}^{\mathrm{C}}{ }_{\mathrm{CR}}$ values in this case. Direct FRET can be separated from sequential FRET by eliminating the YFP fluorochrome in the triple construct. But because YFP could not be eliminated by photobleaching without substantial photobleaching of mRFP, the direct $\mathrm{CFP} \rightarrow$ mRFP transfer efficiency was measured using the CFP-YFP/Y66CmRFP protein $\left(E_{\mathrm{CR}}=0.04\right)($ Fig. 2d). $E$ values obtained for all three direct FRET processes that occur in the CFP-YFP-mRFP protein (Figs. 2c,d) were used to calculate the total amount of energy transferred from CFP directly to YFP and $\mathrm{mRFP}\left(E_{\mathrm{C} \rightarrow \mathrm{YR}}=0.36\right)$, as described in in vitro experiments (Fig. 1). Approximately $9 \%$ of excited CFP energy reaches mRFP, of which approximately one third is due to direct FRET. 
a
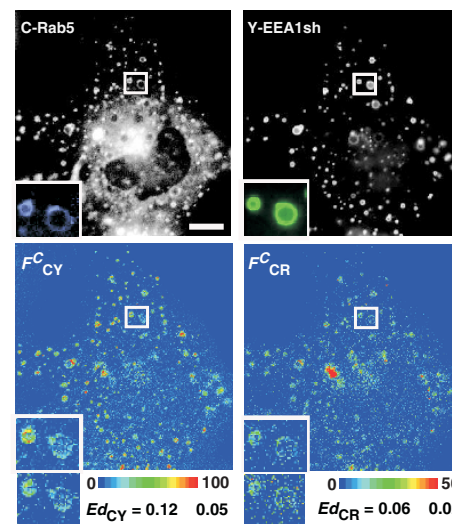

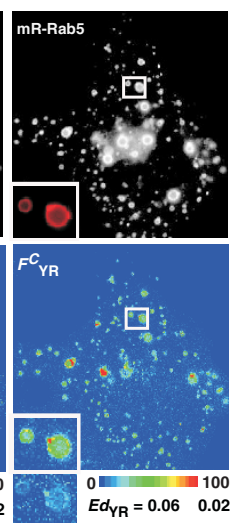

b

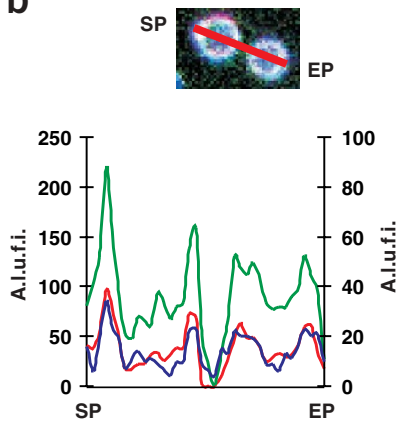

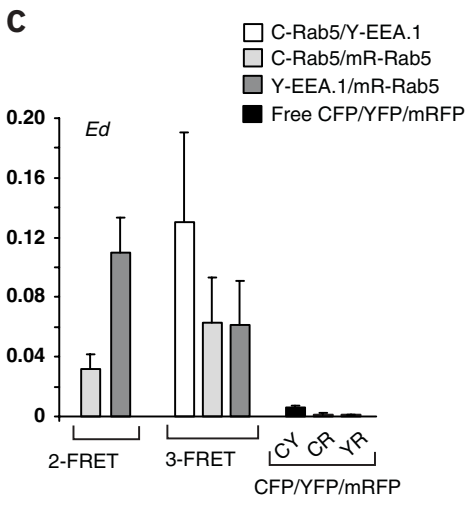

Figure 3 | 3-FRET microscopy analysis of Rab5 microdomains in single endosomes of living cells. (a) mRFP-Rab5, YFP-EEA1.sh and CFP-Rab5 were coexpressed for $2 \mathrm{~d}$, the cells were treated with nocodazole for $15 \mathrm{~min}$, and six images were acquired as described in Figure $2 \mathrm{c}$. FRET $\mathrm{F}^{\mathrm{C}}$ images are presented in pseudocolor mode. Insets show an enlargement of the outlined regions of the images as well as corresponding $E d$ images. Mean $E d$ values measured for individual endosomes of the presented cell are shown above the corresponding image inset. Scale bar, $10 \mu \mathrm{m}$. (b) Three FRET ${ }^{C}$ images in RGB format obtained in 3-FRET experiments (a) (FRET ${ }_{C Y}$ is green; FRET $^{C}$ YR is red; and $F$ RET $^{C}{ }_{C R}$ is blue) were merged. 'White' designates the overlap of red, blue and green. The arbitrary fluorescence intensities of FRET ${ }^{C}$ signals across two endosomes were plotted. FRET $^{C}$ CR is plotted on the right axes. SP is the starting point, and EP is the end point. A.l.u.f.i. is arbitrary linear units of fluorescence intensity. (c) Mean $E d$ values ( \pm s.d.) obtained for single endosomes in cells coexpressing CFP-Rab5 and mRFP-Rab5 or YFP-EEA.1sh and mRFP-Rab5 measured using 2-FRET. Images are shown in Supplementary Figure 2 online and in a (3-FRET). Mean Ed values were also measured for CFPmRFP (CR), CFP-YFP (CY) and YFP-mRFP (YR) FRET pairs using six-filter method in four cells coexpressing free CFP, YFP and mRFP (black bars). The scatter plots of $E d$ values versus acceptor intensity are presented in Supplementary Figure 1 online.

Energy transfer from CFP to YFP and mRFP, and from YFP to mRFP, was confirmed by DFRAP using consecutive photobleaching of mRFP and YFP (Fig. 2e). These experiments were carried out in fixed cells because considerable cell shape changes and redistribution of fluorescent proteins take place in living cells during two rounds of whole-cell photobleaching, which leads to substantial errors in measurements of fluorescence intensities. The FRET efficiencies obtained in experiments with fixed cells were typically lower than those obtained in experiments with living cells owing to reduced quantum yields of CFP and YFP after chemical fixation.

The data in Figure 2 demonstrate that there is good correlation of the FRET efficiencies measured using sensitized acceptor emission and DFRAP methods; that three interdependent FRET processes can be detected using a six-filter method; and that direct $\mathrm{CFP} \rightarrow \mathrm{mRFP}$ FRET can be distinguished from sequential $\mathrm{CFP} \rightarrow \mathrm{YFP} \rightarrow \mathrm{mRFP}$ FRET.

\section{Detection of a three-protein complex}

Whole-cell FRET experiments with fusion proteins demonstrated the feasibility of using mRFP as a FRET acceptor in three-filter and six-filter FRET measurements in vivo. To establish a technique for measurement of 3-FRET between three distinctly tagged proteins in an individual cellular compartment, we constructed mRFP-Rab5 and analyzed a proposed model of protein interactions in endosomal Rab5-early endosomal antigen 1 (EEA.1) microdomains ${ }^{25}$. mRFP-Rab5 was coexpressed with CFP-Rab5 and YFP-EEA.1sh, a fragment of EEA.1 protein (residues 1,306-1,411) that is targeted by its FYVE domain to early endosomes, where it binds to Rab5 (ref. 26). mRFP-Rab5 protein colocalized with both proteins in endosomes (Fig. 3a), suggesting that mRFP tagging of Rab5 does not interfere with Rab5 targeting and function.

To measure 3-FRET, we used the six-filter FRET method to obtain three FRET ${ }^{\mathrm{C}}$ images. To prevent image shifting during sixfilter image acquisition, endosomal movement was reduced by treating cells with nocodazole, an inhibitor of microtubule polymerization. This treatment did not alter cell shape or the morphological appearance of endosomes. FRET $^{\mathrm{C}}$ images indicated three FRET processes: YFP-EEA.1sh $\rightarrow$ mRFP-Rab5, CFP-Rab5 $\rightarrow$ YFP-EEA.1sh, and CFP-Rab5 $\rightarrow$ mRFP-Rab5 (Fig. 3a). Merging three FRET $^{\mathrm{C}}$ images of the individual endosomes produced a similar distribution of FRET ${ }^{\mathrm{C}}$ signals for three FRET pairs across the endosomes, which is consistent with the presence of threeprotein complexes (Fig. $\mathbf{3 b}$ ).

The mean values of apparent energy transfer $(E d)$ calculated for individual endosomes using regional FRET analysis in cells expressing mRFP-Rab5, YFP-EEA.1sh and CFP-Rab5 were significantly $(P<0.02)$ higher than these values in cells coexpressing free YFP, CFP and mRFP proteins (Fig. 3c). Based on measurements of $E d$ values in this negative-control experiment, $E d$ values below 0.01 were considered to indicate the absence of FRET in further experiments. The $E d$ values obtained in regional analysis did not increase with an increase in donor or acceptor concentration in endosomes, suggesting that the observed FRET is not concentration-driven (Supplementary Fig. 1 online).

The data in Figure 3 demonstrate the detection of three-protein complexes in a single compartment of living cells by the 3-FRET method. FRET between CFP-Rab5 and mRFP-Rab5, and between YFP-EEA.1sh and mRFP-Rab5, in endosomes was also confirmed in cells expressing each pair of proteins using sensitized FRET and DFRAP methods (Supplementary Fig. 2 online). In DFRAP experiments using CFP as the donor, a low signal to noise ratio observed for the CFP filter set in the triple-chromophore system resulted in considerable experimental error. Given that the data obtained using sensitized FRET and DFRAP methods correlated well (Fig. 2) and that considerably less variability in $E d$ values was observed in the sensitized FRET measurements, we relied on the sensitized FRET technique in subsequent experiments, using CFP as the FRET donor. 

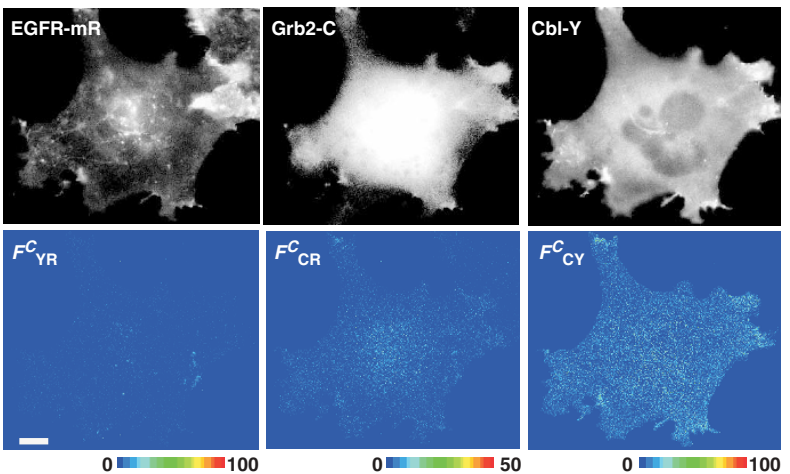

b
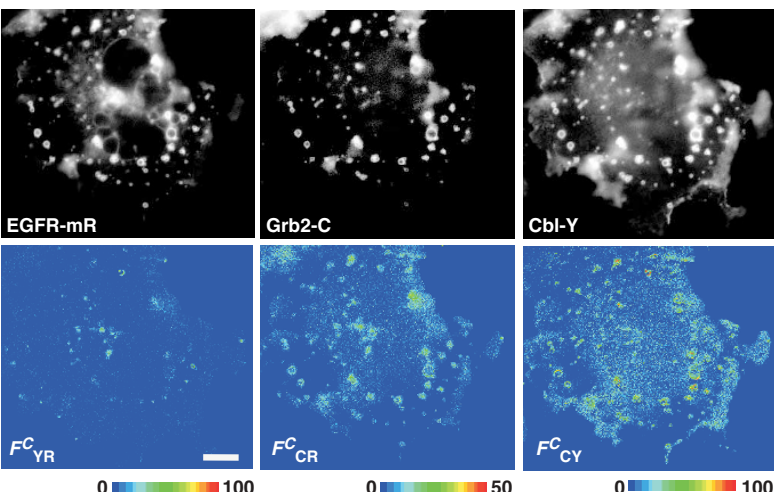

C
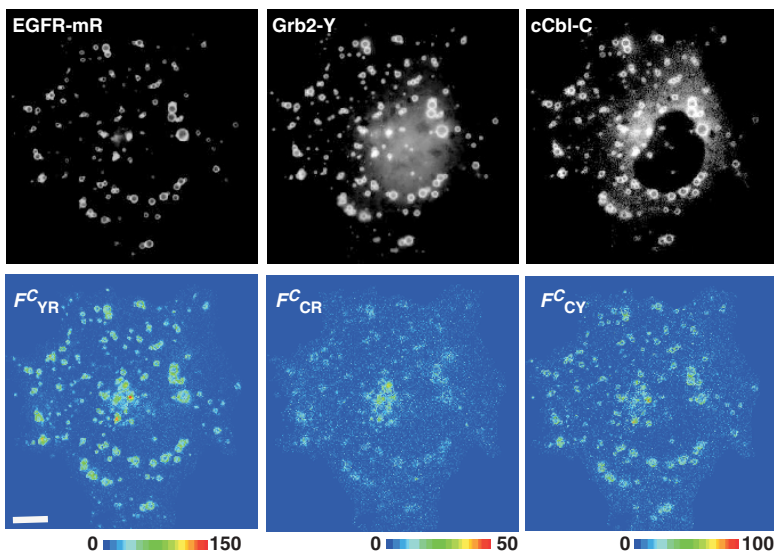

Figure 4 | 3-FRET analysis of EGFR-mRFP interactions with a pair of Cbl-YFP and Grb2-CFP, or a pair of Cbl-CFP and Grb2-YFP $(\mathbf{a}, \mathbf{b})$. EGFR-mRFP was coexpressed with CbL-YFP and Grb2-CFP. Six-filter FRET analysis was carried out on serum-starved cells (a) or cells treated with $17 \mathrm{nM} \mathrm{EGF}$ at $4{ }^{\circ} \mathrm{C}$ and then for $30 \mathrm{~min}$ at $37^{\circ} \mathrm{C}(\mathbf{b})$. The cells were treated with nocodazole that was added for the last 15 min of incubation at $37^{\circ} \mathrm{C}$. (c) EGFR-mRFP was coexpressed with CbL-CFP and Grb2-YFP and six-filter FRET analysis was carried out in cells treated with EGF as described in $\mathbf{b}$. FRET $^{\mathrm{C}}$ images in $\mathbf{a}-\mathbf{c}$ are presented in pseudocolor. Scale bars, $10 \mu \mathrm{m}$.

\section{Analysis of EGFR interactions with Grb2 and $\mathrm{Cbl}$}

We next tested whether 3-FRET can detect stimulus-dependent, dynamic interactions between three different proteins: epidermal growth factor receptor (EGFR), Grb2 and c-Cbl. To this end, a fusion protein of mRFP and EGFR was prepared and transiently expressed in COS-1 cells. The pattern of subcellular distribution of EGFR-mRFP was similar to that previously observed in cells transiently expressing EGFR-GFP ${ }^{27}$. Western-blot analysis
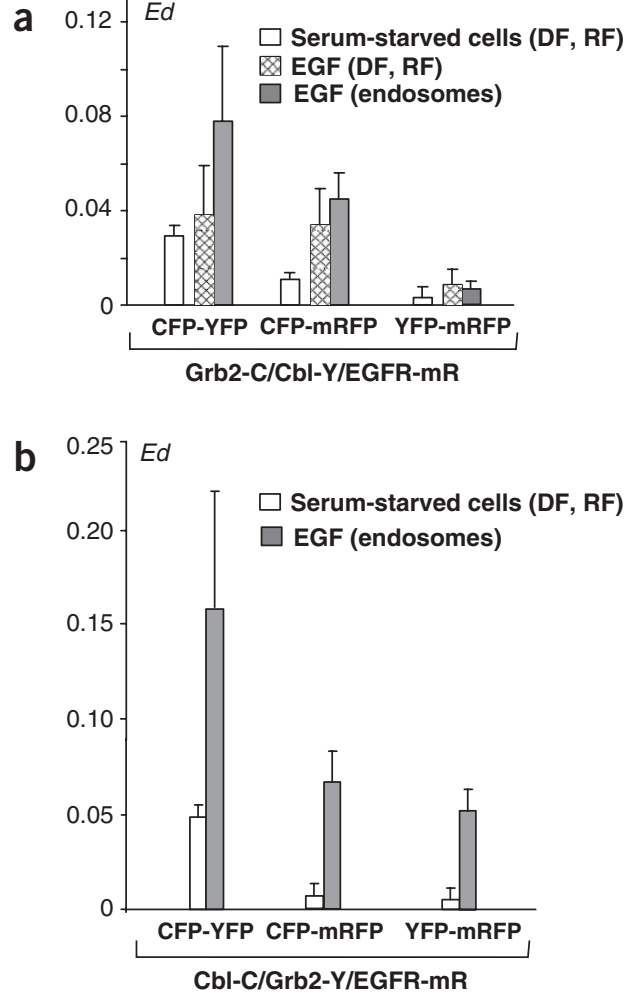

Figure 5 | Comparative 3-FRET analysis of EGFR interactions with differentially tagged $\mathrm{Cbl}$ and $\mathrm{Grb} 2$ proteins. Bar graphs show mean $E d$ values ( \pm s.d.) obtained for diffuse fluorescence areas (DF) and plasma membrane ruffles (RF) in serum-starved untreated cells (white bars) or EGF-treated cells (crosshatched bars), and endosomes of EGF-treated cells (gray bars) in experiments presented in Figure $\mathbf{4 a}, \mathbf{b}(\mathbf{a})$ and Figure $\mathbf{4 c}(\mathbf{b})$. The scatter plots of $E d$ values vs. acceptor intensity are presented in Supplementary Figure 4 online.

demonstrated correct size and EGF-dependent tyrosine phosphorylation of EGFR-mRFP (Supplementary Fig. 3 online).

When EGFR-mRFP was cotransfected with Grb2-CFP and CblYFP, all three fusion proteins were distributed throughout the plasma membrane, cytosol or nucleus (Fig. 4). FRET $^{\mathrm{C}}$ signals calculated for the energy transfer between EGFR-mRFP and Grb2 or Cbl fusion proteins had $E d$ values $\leq 0.01$ (Fig. 5a), comparable to these values obtained for the negative controls (Fig. 3c). Substantial FRET ${ }^{\mathrm{C}}$ signals and $E d$ values were obtained only for the Grb2-CFP-Cbl-YFP pair in the cytosol and ruffle-rich plasma membrane areas, thus confirming the constitutive Grb2-Cbl association $^{28}$ (Figs. 4a and $\mathbf{5 a}$ ).

Upon stimulation of cells expressing the fusion proteins with epidermal growth factor (EGF), strong FRET ${ }^{\mathrm{C}}$ signals and large $E d$ values were obtained for EGFR-mRFP-Grb2-CFP and Grb2-CFP-Cbl-YFP FRET pairs in the plasma membrane, especially in ruffle-rich areas, and endosomes (Figs. $\mathbf{4 b}$ and $\mathbf{5}$, and Supplementary Fig. 4 online). Likewise, substantial FRET ${ }_{C R}{ }_{C R}$ and $E d_{\mathrm{CR}}$ values were detected in endosomes of cells coexpressing EGFR-mRFP and Grb2-CFP in the absence of the Cbl fusion protein (Fig. 6b,c).

In contrast, very minimal, if any, FRET signals were detected between Cbl-YFP and EGFR-mRFP when these proteins were coexpressed with (Figs. 4b and 5a) or without Grb2-CFP, 

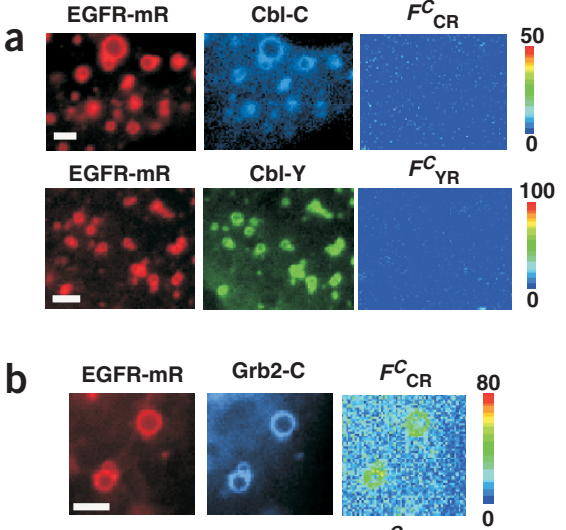

EGFR-mR Grb2-Y
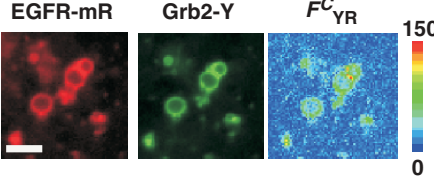

C

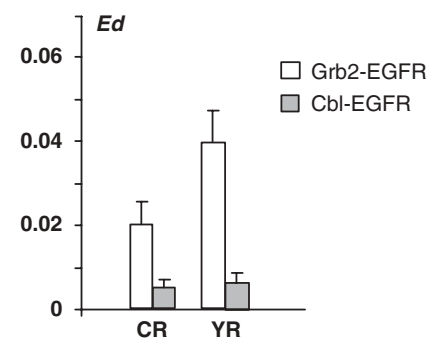

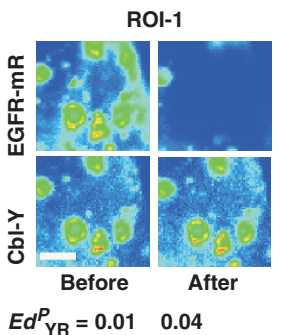

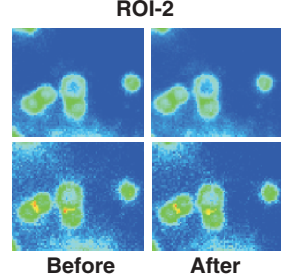

$E d_{\mathrm{YR}}^{P_{\mathrm{R}}}=0.01 \div 0.04$
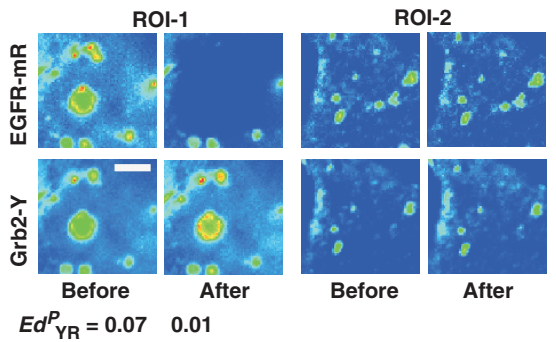

$E d^{P_{\mathrm{YR}}}=0.07 \div 0.01$
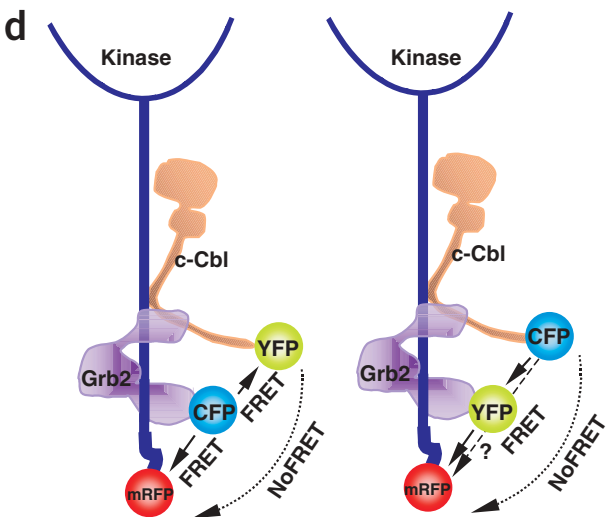

Figure 6 | Two-chromophore FRET and a hypothetic model of EGFR, Grb2 and Cbl interactions. (a) The cells coexpressing EGFR-mRFP with Cbl-CFP or CbL-YFP were treated with EGF as in Figure 4b. 2-FRET measurements and FRET ${ }^{C}$ calculations were carried out as in Figure $2 \mathbf{a}, \mathbf{b}$. Images of representative subregions of cells are shown. For DFRAP experiments, the cells were fixed, and the images were acquired before and after photobleaching of mRFP in a small region of the cell (ROI-1). Images of ROI-1 and another region of the same cell that has not been photobleached (ROI-2) are shown in pseudocolor. The mean $E d^{p}$ values ( \pm s.d.) were calculated for several endosomes in 3-4 cells based on donor dequenching and presented under corresponding images. Scale bars, $3 \mu \mathrm{m}$. (b) 2-FRET and DFRAP experiments were carried out with cells coexpressing EGFR-mRFP with either Grb2-CFP or Grb2-YFP as described in a. (c) Ed values ( \pm s.d) for CFP-mRFP $(C R)$ and YFP-mRFP $(Y R)$ FRET pairs were obtained for multiple single endosomes in EGF-treated cells in experiments presented in $\mathbf{a}$ (gray bars) and $\mathbf{b}$, (white bars). The scatter plots of $E d$ values versus acceptor intensity are presented in Supplementary Figure 4 online. (d) A hypothetical model of one-step, parallel and sequential FRETs within the EGFR-Grb-Cbl complex based on the data of Figures 4-6.
(Fig. 6a) as judged from very low FRET ${ }^{\mathrm{C}}$ and $E d$ values (Fig. 6c). Similar results were obtained with DFRAP experiments (Fig. 6a). No FRET was detected between EGFR-mRFP and YFP-Cbl fusion protein, in which the YFP moiety was attached at the $\mathrm{N}$ terminus of c-Cbl (data not shown). These data suggested that YFP attached to either terminus of $\mathrm{Cbl}$ is unfavorably oriented for direct FRET to the $\mathrm{C}$ terminus of the EGFR (Fig. 6d).

When EGFR-mRFP was coexpressed with Grb2-YFP and CblCFP, substantial FRET ${ }^{C}$ signals and $E d$ values were obtained for the pair of Cbl-CFP and EGFR-mRFP fusion proteins (Figs. 4c and 5b). On the other hand, expression of these two proteins without coexpression of Grb2-YFP yielded no FRET (Fig. 6a,c). Therefore, we hypothesize that FRET from Cbl-CFP to EGFR-mRFP required the presence of the intermediate adaptor, Grb2 tagged with YFP and, therefore, occurred due to sequential $\mathrm{CFP} \rightarrow \mathrm{YFP} \rightarrow \mathrm{mRFP}$ energy transfer (Fig. 6d), as observed for the CFP-YFP-mRFP concatemer (Figs. 1 and 2). Taken together, the data obtained in the 3-FRET analysis provide direct evidence to support the previously proposed model that although $\mathrm{Cbl}$ is capable of direct binding to EGFR, it preferentially binds receptors indirectly through Grb2 (refs. 28,29).

\section{DISCUSSION}

In this study, we characterized CFP-mRFP and YFP-mRFP FRET pairs that utilize mRFP as the acceptor. Because mRFP is a derivative of DsRed, the possibility of spontaneous oligomerization was a concern. For the fusion proteins of EGFR and Rab5 used in this study, no substantial oligomerization was observed under conditions of moderate expression. Fusion of several other proteins with mRFP yielded inactive proteins that exhibited cytoplasmic aggregation. Therefore, further improvement of mRFP is necessary.

When compared for identical fusion proteins, the efficiency of YFP-mRFP FRET was higher than that of the CFP-mRFP pair, regardless of the method of FRET measurement (Figs. 1 and 2). This observation is in good agreement with the shorter Förster distance for the CFP-mRFP pair $\left(R_{\mathrm{CR}}=3.82\right)$, as compared with $R_{\mathrm{YR}}=4.90 \mathrm{~nm}$, estimated for YFP-mRFP (Supplementary Table 1 online). But the minimal cross-bleeding between filter channels used to measure sensitized CFP-mRFP FRET makes this pair useful for FRET microscopy. The $E$ values obtained for CFP-mRFP FRET in cells were lower than the values measured with purified proteins. This discrepancy can be caused by partial degradation of the fusion constructs in vivo ${ }^{30}$, and by the presence of immature nonfluorescent species of mRFP, which absorb at $500 \mathrm{~nm}$ (ref. 17) and therefore act as efficient CFP quenchers. Such CFP quenching is eliminated upon cleavage of the tandem proteins in vitro but not by photobleaching mRFP in cells at $575 \mathrm{~nm}$.

As mRFP was able to serve as a FRET acceptor for CFP and YFP, we expanded the portfolio of FRET techniques with the 3-FRET assay. The experiments with concatemeric proteins demonstrated that, when all three fluorescent proteins (CFP, YFP and mRFP) are in proximity to each other, one-step (YFP $\rightarrow \mathrm{mRFP}$ ), parallel 
$(\mathrm{CFP} \rightarrow \mathrm{YFP}$ and $\mathrm{CFP} \rightarrow \mathrm{mRFP})$ and sequential FRET $(\mathrm{CFP} \rightarrow$ $\mathrm{YFP} \rightarrow \mathrm{mRFP}$ ) can occur and be measured by spectral analysis in vitro (Fig. 1) and using a six-filter microscopic method in living cells (Fig. 2). Calculations of transfer efficiencies using triple fusion proteins correlated well with the theoretical and experimental values obtained for each of the three mutually dependent FRET processes (Fig. 1). E values obtained using a sensitized FRET method and $E^{\mathrm{P}}$ values measured using the DFRAP technique in the same cell were similar, thus confirming the accuracy of calculations of the FRET efficiency using $G$ factors (Fig. 2a).

Development of the six-filter method of intramolecular FRET measurements opened the way to the application of this method to intermolecular 3-FRET analysis in single cellular compartments, which was developed using two experimental models. The first model system of Rab5-EEA.1 microdomains in endosomes is an example of constitutive complexes, where interactions between all three differentially tagged components were detected as three FRET processes (Fig. 3). In the second model, formation of a three-protein complex in response to a stimulus (EGF) was observed through the detection of two direct and one sequential FRET processes (Figs. 4-6). Analysis of the second model showed that the ability to detect the sequential energy transfer by 3-FRET is particularly useful for analyzing ternary complexes in which the interaction between two proteins is mediated by a third molecule.

Rapid movement of organelles, such as endosomes, during sixfilter image acquisition represents a key experimental difficulty in 3-FRET microscopy of living cells. To circumvent this problem, we inhibited microtubule-dependent motility, which markedly reduced endosome movement. Another difficulty in the intermolecular FRET analysis is the quantification of the data. Although FRET $^{\mathrm{C}}$ images offer a qualitative indication of FRET in these experiments, true FRET efficiencies cannot be directly calculated from FRET ${ }^{\mathrm{C}}$ data because of unknown donor-acceptor concentrations and stoichiometry of interactions, as well as the presence of endogenous, unlabeled species of interacting proteins. This raises the issue of what is the best approach to establish the presence or absence of energy transfer from the FRET data and compare FRET data between different samples. The first issue must be addressed by comparing FRET signals in the experimental sample with those in control samples of noninteracting proteins. Although coexpression of nonfused fluorescent proteins is typically used as a negative control (Fig. 3c), the most reliable reference sample of the absence of FRET must be designed so that proteins that are not involved in FRET are expressed at the same levels and have the same pattern of localization in the cell as the experimental pair. For instance, in our studies FRET signals obtained for EGFR-mRFP/Cbl pairs were used as a reference for the absence of FRET, or a minimal FRET, to confirm the presence of FRET in other combinations of EGFR, Grb2 and Cbl fusions, which were present in endosomes in amounts and molar ratios comparable to those in the negativereference samples (Figs. 4-6).

The issue of quantification of intermolecular FRET data has been under much debate $23,31,32$. It has been previously concluded that calculation of the apparent $E$ values ( $E d$ in this paper, equation 2), which roughly represent FRET $^{\mathrm{C}}$ normalized by the donor concentration, results in near-optimal measurement of FRET, and that its subsequent normalization by the acceptor concentration increases the variability in the resulting FRETN values ${ }^{23}$. Moreover, comparison of various methods of calculation of FRET efficiencies in the experimental system of multiple donor-acceptor interactions demonstrated that the method of $E$ calculation $^{23}$ is the most reliable $^{32}$. In our experiments, comparison of $E d$ (equation 2) and FRETN (equation S8 in Supplementary Methods online) values for multiple subregions of the same cell revealed that $E d$ values had the lowest variability (data not shown). We found, however, that it is unpractical to calculate the $E d$ for entire images because of the substantial amplification of the computational noise of low intensity pixels (Fig. 3a). In contrast, calculation of $E d$ values using regional FRET analysis produced much more consistent data because regions or single organelles with no temporal shifts and near-stoichiometric ratios of fluorescent proteins could be selected for calculations.

In conclusion, our studies demonstrate the feasibility of the reliable analysis of dynamic multi-protein assemblies in vivo using the 3-FRET method when it is carried out by careful comparison of FRET $^{\mathrm{C}}$ images and $E d$ values obtained in different experiments with the same donor-acceptor pair of fluorescently tagged proteins that exhibit similar subcellular localization and are expressed at comparable levels.

\section{METHODS}

Plasmids, protein expression and spectroscopy. Construction of bacterial and mammalian expression plasmids, protein expression and purification are described in Supplementary Methods online. Excitation and emission spectra of purified fluorescent proteins and their concatemers before and after cleavage by factor $\mathrm{X}_{\mathrm{a}}$ were measured at $25{ }^{\circ} \mathrm{C}$ in a $0.5-\mathrm{cm}$ quartz cuvette using a PTI Fluorescence Spectrophotometer (Photon Technologies). To minimize cross-excitation of the acceptors in FRET measurements (see Supplementary Methods online), CFP and YFP donors were excited at $420 \mathrm{~nm}$ and $470 \mathrm{~nm}$, respectively.

FRET microscopy. COS-1 cells were transfected using Effectene (Qiagen). Expression of mRFP-fused proteins was confirmed by Western blotting, as described ${ }^{26}$. For microscopy, cells were replated $1 \mathrm{~d}$ after transfection onto $25-\mathrm{mm}$ glass coverslips and incubated in serum- and phenol red-free medium containing $0.1 \%$ bovine serum albumin for $6-20 \mathrm{~h}$ before an experiment. In experiments with EGFR, the cells were stimulated with $17 \mathrm{nM}$ EGF for $30 \mathrm{~min}$ at $4{ }^{\circ} \mathrm{C}$ to allow for maximal occupancy of receptors, and then incubated at $37^{\circ} \mathrm{C}$ for $30 \mathrm{~min}$. Coverslips with cells were mounted in a microscopy chamber and imaged at room temperature. Before image acquisition, cells were treated with $20 \mu \mathrm{g} \mathrm{ml}^{-1}$ nocodazole (Sigma) for $15 \mathrm{~min}$ at $37^{\circ} \mathrm{C}$.

The fluorescence imaging Marianas workstation (Intelligent Imaging Innovation) based on an inverted Zeiss microscope equipped with independently-controlled excitation and emission filter wheels and a micropoint FRAP system (Photonic Instruments) was used.

The method of sensitized two-chromophore FRET measurement has been described previously ${ }^{7,23}$. For 3-FRET, images were acquired sequentially through FRET $_{\mathrm{CY}}, \mathrm{FRET}_{\mathrm{CR}}, \mathrm{FRET}_{\mathrm{YR}}, \mathrm{CFP}$, YFP and mRFP filter channels (see Supplementary Table 2 online) using $2 \times 2$ binning mode and $100-\mathrm{ms}$ to $500-\mathrm{ms}$ integration times. Background for each cell expressing fluorescent proteins was determined as mean intensity of adjacent cells not expressing fluorescent proteins and subtracted from the raw image before carrying out FRET calculations. 
FRET $^{\mathrm{C}}$ was calculated for each of three two-chromophore FRET pairs in the presence of three fluorescent proteins on a pixel-by-pixel basis for the entire image as described for twochromophore $\mathrm{FRET}^{7}$ using the formula shown in equation 1 in a modified notation ${ }^{23}$ :

$$
\mathrm{FRET}^{\mathrm{C}}=F f-D f(F d / D d)-A f(F a / A a)-T f(F t / T t)
$$

$D f, A f$ or $T f$ is the fluorescence signal using donor, acceptor or a third fluorochrome filter channel, respectively, in the presence of three fluorochromes; $F f$ is the fluorescence signal through the FRET filter channel in the presence of three fluorochromes; and $F d / D d, F a / A a$ and $F t / T t$ are cross-bleed coefficients measured in cells expressing only a donor, an acceptor or a third fluorochrome, respectively, as the fraction of the donor, acceptor or the third fluorochrome fluorescence passing through the FRET channel. These coefficients are presented in Supplementary Table 2 online. The $F t / T t$ coefficients, as well as cross-bleeds between CFP, YFP and mRFP filter channels, were found to be effectively zero $(<0.5 \%)$. equation 1 was, therefore, simplified to:

$$
\mathrm{FRET}^{\mathrm{C}}=F f-D f(F d / D d)-A f(F a / A a)
$$

$F d / D d$ and Fa/Aa coefficients were slightly overestimated by rounding up to the nearest hundredth, which could result in underestimation of FRET ${ }^{\mathrm{C}}$ and negative FRET $^{\mathrm{C}}$ pixel values for the control FRET between pairs of noninteracting proteins. Because 'negative' fluorescence intensity does not have physical meaning, for calculation of FRETC images using 'whole-image math', pixels with negative FRET $^{\mathrm{C}}$ intensities were assigned a fluorescence intensity of zero. All images were inspected for shifts of fluorescent compartments during image acquisition and were discarded if such shifts occurred.

In experiments with concatemeric constructs (donor-acceptor 1:1), FRET efficiency $E$ was calculated according to ref. 23 as:

$$
\mathrm{E}=\mathrm{FRET}^{\mathrm{C}} /\left(D f \times \mathrm{G}+\mathrm{FRET}^{\mathrm{C}}\right)
$$

$\mathrm{G}$ is the factor relating the loss of donor signal due to FRET with the donor filter set to the increase of the acceptor emission through the FRET filter set due to FRET $^{23}$. The values of $\mathrm{G}(\mathrm{cy})=3.099, \mathrm{G}(\mathrm{cr})=1.290$ and $\mathrm{G}(\mathrm{yr})=0.416$ were calculated for CFP-YFP, CFP-mRFP and YFP-mRFP donor-acceptor pairs, respectively, as described ${ }^{23}$.

In experiments where the stoichiometry of donor and acceptor interactions was unknown, mean donor, acceptor and FRET ${ }^{\mathrm{C}}$ signals were calculated for several selected subregions of the image containing individual endosomes, ruffles or diffuse fluorescence areas. The $E d$ for each subregion was calculated according to equation 2. $E d$ values were calculated using FRET $^{\mathrm{C}}$ images that were obtained using 'whole-image math' as described for equation 1A. Therefore, mean FRET ${ }^{\mathrm{C}}$ intensities and $E d$ values of subregions were $\geq 0$.

In samples with variable donor-acceptor stoichiometry, $E d$ is an apparent FRET efficiency and cannot be directly related to the true transfer efficiency $E$. Because $E d$ value varies greatly with the significant changes of acceptor-donor ratio, only subregions, where donor and acceptor molar ratios did not differ from each other by more than twofold, were used for calculations of $E d$ values. The relative apparent fluorescence intensities of CFP, YFP and mRFP were calculated using concatemeric constructs in DFRAP experiments. The acceptor intensity was measured before photobleaching, whereas the intensity of the donor was measured in the same cells after acceptor photobleaching. These calculations yielded mean values of the arbitrary units of fluorescence intensity of 1.0:14.1:7.0 for equimolar quantities of CFP, YFP and mRFP, respectively. The method of regional analysis allowed us to avert overestimation and underestimation of FRET signals due to temporal shifts of organelles during image acquisition by exclusively selecting for calculations the organelles that did not shift.

FRET $^{\mathrm{C}}$ and $E$ images are presented in a quantitative pseudocolor mode as described ${ }^{26}$.

DFRAP experiments. Typically, three or six images were acquired in two-chromophore FRET or 3-FRET experiments, respectively, before and after photobleaching of mRFP or YFP using a dye laser at 575 or $515 \mathrm{~nm}$, respectively, either in whole living cells expressing concatemeric constructs or in a region of interest (ROI-1; individual compartments in living cells or subregions in fixed cells). In these experiments, the excitation intensity during image acquisition was reduced due to the introduction of a 50/50 dichromatic for the FRAP system to achieve simultaneous photobleaching and image acquisition. Usually, reduction of the initial mRFP or YFP fluorescence intensity down to a level of $10-20 \%$ was observed after one round of photobleaching without significant reduction of the donor intensities, as tested with cells only expressing the donor. Mean fluorescence intensity of the acceptor and donor in ROI-1 before and after photobleaching was measured. Because of possible endosome movement during photobleaching, careful selection of all pixels corresponding to an endosome was carried out independently on the image before and after bleaching to measure the fluorescence intensities of the whole endosome. To control for cell movement and fluorescence fluctuations independent of bleaching, mean fluorescence intensity of unbleached regions of the cell (ROI2 ) was measured. The experiments, in which minimal changes in ROI-2 occurred, were used for calculations of FRET efficiencies. Mean fluorescence intensities in ROI- 1 and ROI- 2 were measured under the same condition for each data set. All experiments were carried out under identical microscope settings for the laser energy input and the fluorescence detection. The FRET efficiency $E$ was calculated as described ${ }^{32}$ :

$$
E^{\mathrm{P}}=1-\left[\left(D f_{(\mathrm{ROI}-1)} / D d_{(\mathrm{ROI}-1)}\right)\left(D f_{a(\mathrm{ROI}-2)} / D f_{b(\mathrm{ROI}-2)}\right)\right]
$$

$D f_{(\mathrm{ROI}-1)}$ and $D d_{(\mathrm{ROI}-1)}$ are the mean fluorescence intensities of the donor in ROI-1 before and immediately after photobleaching, respectively. $D f_{b}(\mathrm{ROI}-2)$ and $D f_{a(\mathrm{ROI}-2)}$ are the mean fluorescence intensities of the donor in the ROI-2 before and immediately after photobleaching, respectively.

Note: Supplementary information is available on the Nature Methods website.

\section{ACKNOWLEDGMENTS}

We thank R. Tsien and R. Campbell for the gift of mRFP1 cDNA and C. Monks for critical reading of the manuscript. This work was supported by grants from National Cancer Institute and National Institute of Drug Abuse (to A.S. and V.V.) and a postdoctoral fellowship from American Heart Association (to E.G.).

\section{COMPETING INTERESTS STATEMENT}

The authors declare that they have no competing financial interests. 


\section{Received 11 August; accepted 6 0ctober 2004}

\section{Published online at http://www.nature.com/naturemethods/}

1. Gaits, F. \& Hahn, K. Shedding light on cell signaling: interpretation of FRET biosensors. SCi. STKE 2003, PE3 (2003).

2. Jares-Erijman, E.A. \& Jovin, T.M. FRET imaging. Nat. Biotechnol. 21, 1387-1395 (2003).

3. Zhang, J., Campbell, R.E., Ting, A.Y. \& Tsien, R.Y. Creating new fluorescent probes for cell biology. Nat. Rev. Mol. Cell Biol. 3, 906-918 (2002).

4. Chiu, V.K. et al. Ras signalling on the endoplasmic reticulum and the Golgi. Nat. Cell Biol. 4, 343-350 (2002).

5. Haj, F.G., Verveer, P.J., Squire, A., Neel, B.G. \& Bastiaens, P.I. Imaging sites of receptor dephosphorylation by PTP1B on the surface of the endoplasmic reticulum. Science 295, 1708-1711 (2002).

6. Jiang, X. \& Sorkin, A. Coordinated traffic of Grb2 and Ras during epidermal growth factor receptor endocytosis visualized in living cells. Mol. Biol. Cell 13, 15221535 (2002).

7. Sorkin, A., McClure, M., Huang, F. \& Carter, R. Interaction of EGF receptor and grb2 in living cells visualized by fluorescence resonance energy transfer (FRET) microscopy. Curr. Biol. 10, 1395-1398 (2000).

8. Zhang, J., Ma, Y., Taylor, S.S. \& Tsien, R.Y. Genetically encoded reporters of protein kinase A activity reveal impact of substrate tethering. Proc. Natl. Acad. Sci. USA 98, 14997-15002 (2001).

9. Mochizuki, N. et al. Spatio-temporal images of growth-factor-induced activation of Ras and Rap1. Nature 411, 1065-1068 (2001).

10. Violin, J.D., Zhang, J., Tsien, R.Y. \& Newton, A.C. A genetically encoded fluorescent reporter reveals oscillatory phosphorylation by protein kinase $\mathrm{C}$. J. Cell Biol. 161, 899-909 (2003).

2 11. Ohba, Y., Kurokawa, K. \& Matsuda, M. Mechanism of the spatio-temporal regulation of Ras and Rap1. EMBO J. 22, 859-869 (2003).

12. Kraynov, V.S. et al. Localized Rac activation dynamics visualized in living cells. Science 290, 333-337 (2000).

13. Matz, M.V. et al. Fluorescent proteins from nonbioluminescent Anthozoa species. Nat. Biotechnol. 17, 969-973 (1999).

14. Labas, Y.A. et al. Diversity and evolution of the green fluorescent protein family. Proc. Natl. Acad. Sci. USA 99, 4256-4261 (2002).

15. Gurskaya, N.G. et al. GFP-like chromoproteins as a source of far-red fluorescent proteins. FEBS Lett. 507, 16-20 (2001).

16. Verkhusha, V.V. \& Lukyanov, K.A. The molecular properties and applications of Anthozoa fluorescent proteins and chromoproteins. Nat. Biotechnol. 22, 289-296 (2004).

17. Campbell, R.E. et al. A monomeric red fluorescent protein. Proc. Natl. Acad. Sci. USA 99, 7877-7882 (2002).
18. Wouters, F.S., Verveer, P.J. \& Bastiaens, P.I. Imaging biochemistry inside cells. Trends Cell Biol. 11, 203-211 (2001).

19. Watrob, H.M., Pan, C.P. \& Barkley, M.D. Two-step FRET as a structural tool. J. Am Chem. Soc. 125, 7336-7343 (2003).

20. Ohya, Y., Yabuki, K., Hashimoto, M., Nakajima, A. \& Ouchi, T. Multistep fluorescence resonance energy transfer in sequential chromophore array constructed on oligo-DNA assemblies. Bioconjug. Chem. 14, 1057-1066 (2003).

21. Liu, J. \& Lu, Y. FRET study of a trifluorophore-labeled DNAzyme. J. Am. Chem. Soc. 124, 15208-15216 (2002)

22. Pollok, B.A. \& Heim, R. Using GFP in FRET-based applications. Trends Cell Biol. 9, 57-60 (1999).

23. Gordon, G.W., Berry, G., Liang, X.H., Levine, B. \& Herman, B. Quantitative fluorescence resonance energy transfer measurements using fluorescence microscopy. Biophys. J. 74, 2702-2713 (1998).

24. Wouters, F.S., Bastiaens, P.I., Wirtz, K.W. \& Jovin, T.M. FRET microscopy demonstrates molecular association of non-specific lipid transfer protein (nsLTP) with fatty acid oxidation enzymes in peroxisomes. EMBO J. 17, 7179-7189 (1998).

25. de Renzis, S., Sonnichsen, B. \& Zerial, M. Divalent Rab effectors regulate the sub-compartmental organization and sorting of early endosomes. Nat. Cell Biol. 4, 124-133 (2002).

26. Galperin, E. \& Sorkin, A. Visualization of Rab5 activity in living cells by FRET microscopy and influence of plasma-membrane-targeted Rab5 on clathrindependent endocytosis. J. Cell Sci. 116, 4799-4810 (2003).

27. Carter, R.E. \& Sorkin, A. Endocytosis of functional epidermal growth factor receptor-green fluorescent protein chimera. J. Biol. Chem. 273, 35000-35007 (1998).

28. Jiang, X. \& Sorkin, A. Epidermal growth factor receptor internalization through clathrin-coated pits requires Cbl RING finger and proline-rich domains but not receptor polyubiquitylation. Traffic 4, 529-543 (2003).

29. Jiang, X., Huang, F., Marusyk, A. \& Sorkin, A. Grb2 regulates internalization of EGF receptors through clathrin-coated pits. Mol. Biol. Cell 14, 858-870 (2003).

30. Kohl, T., Heinze, K.G., Kuhlemann, R., Koltermann, A. \& Schwille, P. A protease assay for two-photon crosscorrelation and FRET analysis based solely on fluorescent proteins. Proc. Natl. Acad. Sci. USA 99, 12161-12166 (2002).

31. Xia, Z. \& Liu, Y. Reliable and global measurement of fluorescence resonance energy transfer using fluorescence microscopes. Biophys. J. 81, 2395-2402 (2001)

32. Berney, C. \& Danuser, G. FRET or no FRET: a quantitative comparison. Biophys. J. 84, 3992-4010 (2003). 